\title{
Epistemología de la historia de vida en la investigación cualitativa
}

\section{Epistemology of the history of life in qualitative research}

\author{
Andrea Vidanovic Geremich \\ Universidad Iberoamericana del Ecuador-UNIB.E, Ecuador \\ Luis Alfredo Osorio Gómez \\ U.E P. Francisco Febres Cordero- La Salle, Ecuador
}

Autor para correspondencia: andreavidagere@gmail.com, luis_osoriouc@hotmail.com Fecha de recepción: 18 de Noviembre de 2017 - Fecha de aceptación: 15 de Mayo de 2018

Resumen: El proceso de conocer desde el ámbito de la ciencia ha variado en sus formas de abordar los fenómenos desde la epistemología, estableciendo en el tiempo diversos métodos dominados por el paradigma positivista y el paradigma postpositivista, este último dando valor al sujeto y a su interacción con otros; desde esta representación, se piensa en una dialéctica entre el conocedor y el sujeto conocido. El actual estudio tiene como propósito, profundizar en la epistemología de la historia de vida en la investigación cualitativa; para ello, se realizó una revisión documental y se consideraron diversos autores que han aportado de forma significativa al enfoque biográfico. Los resultados se concretan: la historia de vida es un método derivado del enfoque biográfico, responde a la orientación cualitativa; este método se centra en la totalidad de vida de un sujeto representada en un relato, dando razón del mundo interno y social del historiador. Dentro de este marco, la historia de vida exige un proceso metodológico que se detalla en los siguientes pasos: inserción y selección de los sujetos, producción, recopilación y trascripción de la historia de vida, así como su interpretación a través de la hermenéutica para identificar los sentidos y significados emergentes. Palabras Claves: epistemología; enfoque biográfico; historia de vida; método; investigación cualitativa

Abstract: The process of knowing from the field of science has varied in its ways of approaching phenomena from epistemology, establishing in time various methods dominated by the positivist paradigm and the postpositivist paradigm, the latter giving value to the subject and their interaction with others; From this representation, we think of a dialectic between the knower and the known subject. The purpose of the present study is to deepen the epistemology of life history in qualitative research; For this purpose, a documentary review was carried out and several authors who have contributed significantly to the biographical approach were considered. The results are specified: the life history is a method derived from the biographical approach, responds to the qualitative orientation; this method focuses on the totality of life of a subject represented in a story, giving reason to the internal and social world of the historian. Within this framework, life history requires a methodological process that is detailed in the following steps: insertion and selection of subjects, production, collection and transcription of life history, as well as its interpretation through hermeneutics to identify the senses and emerging meanings.

Key Words: epistemology; biographical approach; life history; method; qualitative research 


\section{Introducción}

La investigación en las últimas décadas se ha enriquecido considerablemente por el surgimiento de nuevos modos de concebir la realidad. El fortalecimiento del paradigma postpositivista y de la modalidad cualitativa de investigación invitan a los profesionales de las ciencias sociales a considerar nuevos enfoques para la producción del conocimiento científico. Al respecto, Moreno (2002) plantea que al liberarse el postpositivisvo del positivismo, las ciencias humanas comenzaron a manejar nuevos métodos de investigación bajo una visión humanista, en la que prevalece la orientación cualitativa.

El campo de la investigación cualitativa se interesa por entender los fenómenos humanos, desde la óptica del propio actor. De ahí que los datos recabados durante el proceso, consten de ricas descripciones sobre la realidad estudiada Kavale (como se citó en Charriez, 2012). Esta postura destaca el valor que tiene el sujeto o los sujetos inmersos en la investigación cualitativa en el momento que ponen de manifiesto con sus acciones, verbalizaciones, creencias, vivencias, emociones, conductas, el constructo social que se encuentra en estudio, pues no es la visión del investigador lo que prevalece, es la mirada de los propios sujetos en estudio, quienes encauzan el proceso de interpretación y los hallazgos que se produzcan en el mismo. En esta dirección, Denzin y Lincoln (2012) manifiestan que los investigadores cualitativos intentan dar sentido o interpretar los fenómenos de acuerdo al significado que las personas les otorgan.

En este sentido, el sujeto o los sujetos en estudio pasan a ser agentes activos y los principales protagonistas en el proceso de la investigación cualitativa, quienes aportan una manera particular de concebir su propia realidad en el marco de su entorno cotidiano o natural, aspectos vitales para el investigador. Según Denzin y Lincoln (2012), esta perspectiva de investigación es naturalista, interpretativa y multimetódica; características que hacen referencia a que los datos se obtienen del lugar donde se presenta y desenvuelve el fenómeno; a la manera en que se procesa la información, cuya acción fundamental es la interpretación; y, sobre los diversos métodos que existen para producir el conocimiento científico, ya que son varios y el uso de cada uno depende del fenómeno que se estudia.

Otros autores como Ferrarotti (2011), enfocan la importancia que tiene el ambiente naturalista en la investigación cualitativa, al destacar que, las áreas problemáticas y los conceptos operativos no deben generarse en el interior de una oficina, sino a través de un proceso inductivo de investigación sobre la base de una exploración preliminar. Este autor añade un aspecto elemental, como lo es el proceso inductivo que se debe seguir en la investigación cualitativa, el cual va de lo particular a lo general, de las partes al todo, del sujeto o grupo de sujetos a un entramado social y de un fenómeno singular a teorías de trascendencia en el campo científico.

Ahora bien, en el abordaje de los fenómenos sociales, lo cualitativo no sustituye lo cuantitativo, cada uno tiene sus bondades y sentido en el campo del saber; sin embargo, el modelo cualitativo se ha configurado como una nueva opción que se caracteriza por ser flexible, holística, dialéctica, inductiva, comprensiva y profunda al momento de estudiar las realidades humanas y generar aportes científicos a las ciencias sociales. Martínez (como se citó en Moreno, 2006) fundamenta este aspecto, al plantear que "la investigación cualitativa trata de identificar la naturaleza profunda de las realidades, su estructura dinámica, aquella que da razón plena de su comportamiento y manifestaciones" (p. 11). 
Por otra parte, la condición multimetódica de la investigación cualitativa y el avance que ha tenido en las últimas décadas, debido a los aportes de la sociología, antropología, psicología y filosofía han permitido el surgimiento de diversos enfoques para alcanzar la compresión profunda de las realidades humanas. Entre ellos, se encuentra el biográfico, que de acuerdo a Cornejo (2006), trasciende a un método o técnica de investigación y busca posicionarse como un enfoque, pues incorpora una nueva concepción de lo humano, del sujeto de investigación, de la realidad social, de las posibilidades de conocerla y de los métodos propicios para tal fin.

En este orden de ideas, existen diatribas en cuanto al término que se utiliza para designar a lo biográfico en el marco de la investigación cualitativa. Los autores lo asumen como un método, una perspectiva o un enfoque. Ante este particular Bertaux (como se citó en Betaux 1999) plantea lo siguiente:

Pero, ¿por qué hablar de enfoque biográfico y no de "método de relatos de vida"? La expresión enfoque biográfico constituye una apuesta sobre el futuro. Expresa una hipótesis, a saber, que el investigador que empieza a recolectar relatos de vida creyendo quizás utilizar una nueva técnica de observación en el seno de marcos conceptuales y epistemológicos invariables, se verá poco a poco obligado a cuestionarse estos marcos uno tras otro. Lo que estaría en juego no sería sólo la adopción de una nueva técnica, sino también la construcción paulatina de un nuevo proceso sociológico, un nuevo enfoque que, entre otras características, permitiría conciliar la observación y la reflexión. De allí el término enfoque biográfico. (p. 3).

La presente reflexión que realizó Bertaux hace varios años, planteando la posibilidad del surgimiento de un nuevo proceso sociológico o enfoque que permitiría conciliar la observación y la reflexión; en la actualidad es un hecho consolidado. Se ha estructurado un modelo de investigación autónomo, basado tanto en las experiencias de vida de los sujetos, como en el significado que las mismas tienen para ellos; donde resulta clave el entorno y los diversos métodos, técnicas e instrumentos que se utilizan para la develación de los fenómenos sociales con el rigor científico correspondiente. Todos estos elementos, configuran el nuevo enfoque biográfico arraigado a la literatura contemporánea de la investigación cualitativa.

El enfoque biográfico, a partir del testimonio recolectado de los sujetos sobre sus experiencias y el significado de las mismas, logra establecer una relación entre cada uno y sus contextos para poder profundizar en la realidad que muestran los sucesos narrados. De esta forma, es posible comprender el rumbo histórico de las personas, sus experiencias, visión del mundo, sus aspiraciones a futuro y preferencias; pero también la realidad social donde se encuentran inmersas. Según Jones (como se citó en Chárriez, 2012), de todos los enfoques vinculados a la investigación cualitativa, quizás éste sea el que mejor permita a un investigador conocer cómo las personas asumen y reflejan el contexto social que les rodea; dicho en otras palabras, el enfoque trasciende a la simple comprensión del sujeto, pues facilita a su vez, la interpretación de su núcleo de relaciones y de su entorno.

En cuanto a la definición de enfoque biográfico, Denzin (como se citó en Bisquerra, 2004) lo asume como el estudio, utilización y recopilación de documentos personales durante el proceso de investigación, tales como: autobiografías, biografías, diarios, cartas, historias de vida y relatos de vida. De la misma forma, Bisquerra (2004) destaca que las comunidades, las culturas y las expresiones de la experiencia humana pueden manifestarse en representaciones simbólicas 
orales o escritas que se plasman en los documentos personales. Aunque estos autores consideran la historia de vida y otros elementos del enfoque biográfico como documentos personales que sirven para el estudio, registro y compresión de las realidades humanas, más adelante se destacarán todos los aspectos necesarios para argumentar la concepción de la historia de la vida como método de investigación.

\section{Método}

La presente investigación es de tipo documental porque todos los hallazgos se produjeron a partir de la indagación bibliográfica, que de acuerdo a Arias (2012) consiste en la revisión, sistematización, análisis, contrastación e interpretación de datos generados y reportados por otros investigadores en fuentes documentales, las cuales pueden ser impresas o digitales. En este sentido, se realizó una búsqueda a fondo de los autores que en los últimos años han aportado de forma significativa al enfoque biográfico, específicamente a la historia de vida como método de investigación cualitativa; tal es el caso de Franco Ferrarotti, Daniel Bertaux y Alejandro Moreno. De igual manera, se revisaron artículos científicos y libros de otras personalidades que han contribuido a la comprensión de este fenómeno.

Algunos autores, como es el caso de Palella y Martins (2012) reseñan, que cuando se opta por investigaciones documentales, el responsable de la misma, genera resultados coherentes, descriptivos y significativos según las fuentes bibliográficas consultadas. Esta afirmación, denota el nivel de profundidad descriptivo asociado al presente estudio, cuyo propósito, según Hernández, Fernández y Baptista (2014) consiste en "especificar propiedades y características importantes de cualquier fenómeno que se analice" (p. 92). De forma concreta, se asume este nivel de profundidad porque se pretende desentrañar con detalle, la esencia o las propiedades existentes en la epistemología de la historia de vida.

\section{Resultados y discusión}

\section{La Historia de vida como método de investigación}

La historia de vida involucra la totalidad de la vida de un sujeto, revela su esencia y las múltiples estructuras que en ella coexisten, Ferrarotti (2007) dice: "es algo "vivido": con un origen y un desarrollo, con progresiones y regresiones, con contornos sumamente precisos, con sus cifras y su significado" (p.28). Es decir, todo lo concerniente a las experiencias de vida, en un tiempo pasado con toda la significación y matices de los hechos que han trascendido en el sujeto.

La historia de vida se refiere a una narración de la propia vida de un sujeto a otra persona física, que figura como interlocutor o sujeto investigador (Moreno, 2006), esta narración es profunda, asociada a las prácticas y al sentido que el sujeto les haya otorgado a sus vivencias (Chárriez, 2012). Al respecto, Ferrarotti (1990) añade que se trata de un testimonio directo de todo un período de existencia. En otras palabras, la historia de vida consiste en las explicaciones del protagonista sobre sus acciones, relato que evidencia el campo fenoménico del sujeto.

En este orden de ideas, el protagonista refleja su experiencia subjetiva, Chárriez (2012) indica que "las historias de vida conforman una perspectiva fenomenológica, la cual visualiza la conducta humana, lo que las personas dicen y hacen, como el producto de la definición de su 
mundo" (p.53). Es el mundo interno del sujeto lo que queda revelado en una historia de vida, propósito que se alcanza mediante la relación interpersonal sujeto e investigador.

En la historia de vida, brotan los hechos impregnados de significados y se construyen a partir de las experiencias del sujeto, formando en él una apreciación de su propia vida, su mundo, su yo, y su realidad social (Chárriez, 2012). El yo se edifica en base a la realidad vivida por el sujeto, donde el convivir (vivir con otros) es un elemento interactuante en dicha construcción, que involucra emociones, sentimientos, experiencias, logros, fracasos, frustraciones entre otros; un sin número de significados que el sujeto refleja en la narración de su historia.

Así mismo, la historia de vida envuelve los acontecimientos experimentado por el sujeto con respecto a: escolaridad, salud, familia, amigos, entre otros (Chárriez, 2012), el sujeto exterioriza las relaciones de su entorno social. De esta manera, "las historias de vida ofrecen un marco interpretativo a través del cual el sentido de la experiencia humana se revela en relatos personales de modo que da prioridad a las explicaciones individuales" Jones (como se citó por Chárriez 2012, p.52). Cabe destacar, que el relato de una vida permite percibir, interpretar y comprender el mundo de significado que se haya en la experiencia humana y revela el marco referencial del sujeto.

Por consiguiente, las historias de vida se constituyen de "relatos que se producen con una intención: elaborar y transmitir una memoria personal o colectiva que hace referencia a las formas de vida" Santamarina (como se citó Chárriez, 2012, p.54). En este contexto, desde una historia de vida se pretende interpretar el trasfondo existencial de la vida de un sujeto; evidenciada en todos los recuerdos sobre los acontecimientos vividos, que al ser expresados oralmente muestran la carga significativa que tienen en la construcción del yo. Adicionalmente, los recuerdos dejan ver la memoria colectiva, concentrada en signos, creencias, prácticas, tradiciones, entre otros; que habla del entorno y los grupos directamente relacionados con el protagonista de la historia.

La Historia de vida está constituida por una red de relaciones sociales tejida por la vida misma del grupo humano, en la que el investigador se interesa y pertenece de alguna forma a ese tejido. Córdova (1990) define a las historias de vida como:

Una metodología que no se apoya en procedimientos de carácter estadístico, de carácter muestral, sino que, por el contrario, reivindica un aspecto importante del conocimiento social que es la propia experiencia humana, la propia subjetividad como fuente de conocimiento y de relato de los distintos autores, ya sea de procesos sociales, de elementos puntuales o fenómenos sociales que sirven de correlato para construir el conocimiento de lo social. (p.10).

Por ello, las historias de vida describen la cotidianidad del ser humano, accede a comprender el todo en el cual intervienen múltiples elementos como: biológico, psicológico, social, cultural, económico, educacional, profesional entre otros, del individuo. Son relatos que parten de la realidad y, como método, busca estudiar la experiencia humana, pues el relato de las personas evidencia o deja ver una realidad, constituida por un cúmulo de experiencias desde la infancia hasta el momento de la narración de la historia. 
En este orden de ideas, Taylor y Bogdan (como se citó en Cornejo, Mendoza y Rojas, 2008) señalan que un método es "la manera en que examinamos los problemas y buscamos una solución" (p.97). En la investigación cualitativa, es el fenómeno quien conduce el camino epistemológico y dentro del enfoque biográfico se cumple la misma condición. Dentro de este planteamiento, Moreno (2006) expresa que el investigador debe decidir la terminología a utilizar, sea técnica, método o de otra manera, este aspecto va a depender del sentido que le quiera otorgar el investigador.

Sin embargo, en este desarrollo documental se asume el término "método" porque la historia de vida exige un modo ordenado para interpretar toda una realidad de vida con estructuras, constituidas por conexiones entre diversos elementos, que representan la complejidad humana. Por lo tanto, la historia de vida como método se puede sustentar con el aporte de Moreno (2006) quien la concibe, como una "manera autónoma de investigar con sus propios fundamentos teóricos y sus propios modos de conducir la producción del conocimiento" (p.25). De allí que, la historia de vida cuenta con un camino metodológico interpretativo, adecuado para la comprensión del entramado de las realidades humanas, sustentando sus fundamentos principales en el enfoque biográfico.

\section{La relación sujeto-contexto-investigador en el método historia de vida}

En el enfoque biográfico como se dijo anteriormente y más específicamente en el método historia de vida, se precisa un cambio en la concepción de lo humano, del sujeto de investigación y de la realidad social. Esto a su vez, genera una nueva mirada en las relaciones que se establecen durante el proceso de investigación: relación sujeto en estudio - investigador, relación sujeto en estudio - contexto y relación investigador - contexto. En los siguientes párrafos, se explica en qué consisten estas relaciones desde la óptica del método historia de vida.

En primer lugar, se analiza la relación sujeta en estudio - investigador. De acuerdo a Ferrarotti (2007), la reunión para generar las historias de vida presupone, una relación de confianza entre el sujeto en estudio y el investigador. Esto quiere decir, que la investigación es concebida como una coinvestigación. Dicho análisis, pone de manifiesto la relación cercana que debe existir entre los entes involucrados en el abordaje metodológico de la investigación con historias de vida, donde el sujeto en estudio es visto como otro investigador que aporta y ayuda a comprender el fenómeno, ante el cual, el investigador está obligado a ganarse su confianza. Citando a Ferrarotti (2007): "Entonces, tengo frente a mí una historia de vida, muy bien registrada y, por lo tanto, "desembobinada". La he obtenido a partir de mi interlocutor ganándome su confianza" (p. 27).

En este orden de ideas, de acuerdo a la visión de Ferrarotti, la confianza entre el sujeto en estudio y el investigador es un factor clave dentro del método historia de vida, pues ayuda a que la misma sea completa, profunda, detallada, vinculada al entorno social, pero sobretodo, cargada de significados. Moreno (2006), en sus trabajos, utiliza el término historia-de-vida (con guiones) cuando ésta se estructura con la participación del sujeto en estudio y del investigador como interlocutor real, presente y actuante; de igual manera, asume que la historia de vida generada bajo este esquema, es un producto de dos, es decir, el resultado de la relación que ambos establecen. 
De acuerdo a lo descrito en el párrafo anterior, la investigación se concibe como el producto de la relación, vinculación y cooperación entre el investigador y el sujeto en estudio, donde éste último se cataloga como protagonista. Ferrarotti (1990), señala al respecto, que "el interlocutor no es simplemente un objeto de investigación; es un ser humano que se confía, que te brinda su vida en la mano" (p. 186). Con esta cita, queda reflejada la posición del sujeto en estudio dentro del método historia de vida, la cual trasciende a un objeto de investigación para convertirse en actor principal del proceso, con quien el investigador debe generar confianza, empatía y un vínculo que facilite el trabajo en conjunto para la producción e interpretación de la historia de vida. En palabras de Cornejo (2008), en este método se establece una relación sujeta sujeto que denota su dimensión epistemológica y desemboca en prácticas metodológicas definitorias para este modo de producir conocimiento científico.

En cuanto a la relación sujeto en estudio - contexto, el método historia de vida contempla que toda persona narra su pasado enmarcada en un espacio, tiempo y sociedad determinada, aspectos que influyen considerablemente en su relato. Siguiendo con esta idea, Sanz (2005) reseña que las experiencias pasadas, los modos de vida y el contexto social en el cual un sujeto se encuentra inserto, afectan su comportamiento, su personalidad, su modo de pensar, la narración de su historia personal y la significación que le atribuye a cada una de sus vivencias. En esta explicación, radica la repercusión trascendental que tiene el contexto social en la historia narrada por el sujeto en estudio, lo que se puede concretar de una mejor manera con lo expresado por Ferrarotti (1990) al afirmar que el hombre adquiere una cultura, la aprehende y la internaliza para luego reproducir una síntesis singular de la misma.

En este sentido, el método historia de vida considera que el entorno sociocultural se encuentra reflejado en la persona que narra sus experiencias pasadas. Por lo tanto, no solo permite conocer un individuo, un hecho o una historia aislada; facilita además, la comprensión de toda una familia, comunidad, provincia, país o sociedad en general, de acuerdo al momento histórico que el protagonista haya experimentado. En este particular, Ferrarotti (como se citó Moreno, 2006), recalca que "la historia de vida es la contracción de lo social en lo individual, de lo nomotético en lo idiográfico. En la vida de cada cual está toda su sociedad vivida subjetivamente" (p. 22). En esta misma línea, Santamarina (como se citó en Chárriez, 2012) señala que la historia de vida permite generar y compartir una memoria colectiva o formas de vida de una sociedad en un momento histórico determinado. Estos planteamientos son los que justifican el hecho de conocer y develar toda una sociedad en una sola historia de vida.

Por otro lado, las historias de vida ayudan a descubrir los elementos del entorno, tales como: prácticas sociales, creencias, hechos, acontecimientos, tradiciones, formas de vida, relaciones, entre otros aspectos, llenos de significado para el sujeto en estudio, quien en su narración espontanea como protagonista, dejará fluir de forma natural. Relacionado a esta descripción, Lucca y Berríos, (como citó en Chárriez, 2012) manifiestan que las historias de vida permiten desentrañar las realidades que viven los contextos o sociedades, logrando que lo implícito sea explícito, lo oculto sea visible, lo amorfo tenga forma y lo confuso, sea comprensible. El contexto no puede ser un elemento ajeno al sujeto en estudio, tampoco puede ser ajeno al investigador, es un elemento clave en la investigación con historias de vida, es lo que resulta atractivo para quienes optan por este método cuando deciden comprender algún fenómeno. 
En cuanto a la relación investigador - contexto, queda clara la importancia del entorno en el método historia de vida. El investigador no es un agente ajeno medio que le rodea, debe asignarle la importancia que merece, inmiscuirse en él, describirlo, detallarlo, reflexionarlo y profundizarlo. Esto permitirá esclarecer muchos elementos confusos y a su vez, facilitará la interpretación y comprensión del fenómeno. Varios autores, entre ellos Mallimaci y Giménez (2006) reseñan que, al entrevistar a una persona con el objetivo de desarrollar un estudio con historia de vida, es trabajo del investigador conocer lo más posible sobre el contexto donde el protagonista se desenvuelve y se ha desenvuelto hasta el momento. En esta misma dirección, Moreno et al. (1998) refiere que "la historia del investigador es y debe ser la historia de implicación con la realidad popular" (p. 119). De igual manera, Ferrarotti (2007) exterioriza que el investigador debe "aprehender el nexo entre texto, contexto e intertexto" (p.27).

Todos estos autores con diferentes palabras hacen referencia a la importancia de que el investigador se involucre y/o familiarice con el entorno donde se desenvuelve el sujeto en estudio, lo conozca y lo aprehenda. Incluso, Rodríguez (2003) comenta que el investigador debe trascender a un observador externo o participante para convertirse en un conviviente, donde el grado de inserción y de relación investigador - contexto es más estrecha, continua, cercana y significativa. En síntesis, se debe procurar una compresión del contexto desde adentro, permeando el entorno, involucrándose en el espacio de coexistencia del sujeto en estudio y partiendo de una inserción que valore tanto la confianza, como la relación.

\section{Metodología de Investigación con el Método Historias de Vida}

\section{Inserción y Selección de los Sujetos}

En el proceso de selección de los sujetos, es necesario la inserción del investigador en el campo para conocer el entorno e involucrarse con todos los elementos significantes del mismo, aspectos que facilitan la comprensión de las historias de los informantes y el planteamiento de preguntas precisas que contribuyen a establecer la cercanía con el historiador Atkinson (como se citó en Mallimaci y Giménez, 2006).

La selección de los protagonistas de una historia de vida va a depender del ámbito de la investigación, así que, la cantidad y el tipo de personas entrevistadas no se precisa con detalles al inicio de un estudio; se parte de una idea general de los posibles sujetos de la investigación y pueden ser modificados después de las primeras entrevistas (Taylor y Bogdan, 1987). Cabe añadir, que investigar con historia de vida, implica un proceso de construcción continúo basado en la dialéctica que emerge de la experiencia de los sujetos y la idea de investigación.

En este orden, los protagonistas del estudio tienen que ser representativos para responder potencialmente a los elementos centrales de la investigación. Pues bien, en la selección de los sujetos, se requiere establecer los criterios de inclusión y exclusión, de manera que la elección esté gobernada por la objetividad (Charriez,2012). En efecto, el investigador tiene que contar con una descripción clara y detallada de los aspectos señalados que definen al sujeto como representativo y adecuado para la profundización del estudio. 
Los primeros relatos que se obtienen, producen indicios para la selección del sujeto, pues en esta etapa denominada exploratoria, se opta por las historias que encarnan el fenómeno de estudio (Bertaux, 2005). En este proceso de indagación, el investigador busca un tipo de persona que cumpla con especificidades y experiencias relevantes para la investigación (Taylor y Bogdan, 1987). En términos generales, el o los historiadores demuestran su representatividad a través de significados cónsonos al ámbito de la investigación, los cuales surgen en el dialogo de forma espontánea.

En las historias de vida, el sujeto seleccionado es denominado de varias formas: historiador, co-investigador, protagonista, sujeto en estudio, informante clave entre otros. En cualquiera de los casos, hace referencia a la persona que de forma voluntaria narra su vida con el propósito de apoyar activamente a una investigación. En palabras de Moreno (2006), "narradoractor", es decir, la persona que cuenta su vida, requiere cumplir con algunas particularidades para que sea considerado un narrador efectivo. Entre los aspectos que debe cumplir el narrador para ser característico, son: capacidad expresiva que le permita realizar un relato grato y comprensible, memoria clara y representativa de sus experiencias, sinceridad para dar a conocer hechos reales y no fantasioso, disposición al momento de compartir espacios de encuentros, tomando en consideración el tiempo que exige una historia vida y las sesiones necesarias para profundizar en ella (Pujadas, 2000).

Estos elementos son importantes al momento de elegir un perfil idóneo para el relato de una historia de vida porque a través de ellos, se podrá desentrañar los significados que brotan en las palabras, los recuerdos, gestos, tono de voz, emociones, ritmo de la narración entre otros. Al respecto, Mallimaci y Giménez (2006), agregan: "las personas no poseen los significados, sino que son poseídas por ellos" (p. 8).

Cabe resaltar, que el historiador deberá identificarse con la investigación y ser activo en cada reunión que favorezca al desarrollo de los relatos; se trata de una acción totalmente voluntaria y de interés para el informante clave, por ende, se mostrará comprometido en sus narraciones (Cornejo, Mendoza, y Rojas, 2008). En pocas palabras, el historiador se entrega e involucra en la construcción de la investigación y el vínculo de confianza establecido con el investigador lo lleva a comprometerse en un trabajo continuo, profundo y reflexivo.

Por otro parte, el investigador o co-historiador, es la persona que, partiendo de una idea de investigación, se encarga de seleccionar los historiadores, producir la historia de vida, así como descubrir, interpretar y comprender los significados para generar la teorización. El coinvestigador establece una relación interpersonal con el historiador, donde predomina la entrega y espontaneidad.

\section{Producción de la Historia de Vida}

El proceso de producción de la historia de vida, comienza con la recolección de la información, mediante la técnica de entrevista denominada por diferentes autores como: no directiva, no estructurada, narrativa, abierta, en profundidad, entre otras. La entrevista desde esta perspectiva, valora la escucha del sujeto en estudio, en las diferentes fases de su existencia, Mallimaci y Giménez (2006) expone que la entrevista en profundidad, consiste en "encuentros cara a cara entre el investigador y los informantes, encuentros éstos dirigidos hacia la 
comprensión de las perspectivas que tienen los informantes respecto de sus vidas, experiencias o situaciones, tal como las expresan con sus propias palabras" (p.101).

La entrevista asume un proceso de dialogo, en el cual emerge la totalidad del ser; desde la relación, se piensa en el otro, por ende, es un modo natural de interacción y se desarrolla en el encuentro. Este acto, no contempla preguntas sino temas generales, en los cuales el investigador desea indagar en relación al propósito del estudio para profundizar con detalle en algunos elementos de la historia. Las acciones del investigador radican en impulsar las reflexiones en el historiador con los temas propuestos. Las recopilaciones de los encuentros se dan a través de dos formas: grabando o tomando notas de ellos (Bertaux, 2005). Para captar los detalles de la entrevista, es recomendable sean grabadas en todos los encuentros; estas grabaciones deben ser preferiblemente en audio y video. En cuanto a las notas, se sugieren registrar las observaciones y reflexiones que se generan de ellas.

Seguidamente, se efectúa el proceso de trascripción de la entrevista, Bertaux (2005), le asigna el nombre de: "retranscribir" (p.74). Desde la idea de Cornejo, Mendoza y Rojas (2008), "los relatos son transcritos en su totalidad, conservando en lo posible toda su riqueza en las transcripciones, las faltas de lenguaje, lapsus, titubeos, modismos, así como los silencios o pausas de los narradores en ciertos pasajes del relato" (p.36).

De este modo, en la trascripción se busca reflejar los significados que germinan en cada uno de los detalles concentrados en la historia del sujeto en estudio, que se definen como gestos, movimientos, expresiones faciales, las entonaciones de la voz, las palabras, entre otros. Se invita a ejecutar el proceso de transcripción en las próximas horas después de la recopilación, actividad que va a facilitar el análisis de la historia y a generar nuevos temas de dialogo en los siguientes encuentros.

\section{Interpretación}

En la metodología cualitativa, la historia de vida desencadena un proceso interpretativo que se centra en la esencia de las realidades, expresadas en la narración de la vida de un sujeto, mediante significados simbólicos especificados en el párrafo anterior. Por tal motivo, en el proceso de recolección de la información se debe absorber todos los datos necesarios que favorezcan a la interpretación; de esta forma se va construyendo la línea de vida que se teje en cada experiencia relatada, procedente de la cotidianidad y la recuperación que el individuo mismo hace de su propia vida (Granados, Alvarado y Carmona, 2017).

Por consiguiente, el historiador narra los hechos que para él tienen carga significativa y muestra en ellos el mundo social. En este momento, la interpretación comienza a ganar terreno para comprender una realidad total, por medio de la hermenéutica. Al respecto, Martínez (2006), expresa que la hermenéutica proviene del griego hermeneuein que es "interpretar" cabe decir, descubrir el significado y sentido de los hechos. Por su parte, Dilthey (como se citó Martínez, 2006) define la hermenéutica como: "el proceso por medio del cual conocemos la vida psíquica con la ayuda de signos sensibles que son su manifestación" (p.119). Este autor agrega: "toda expresión de la vida humana es objeto natural de la interpretación- hermenéutica” (p.120). 
Ahora bien, desde la hermenéutica se interpreta toda manifestación de la vida del sujeto y en la historia de vida se persigue este propósito a través del texto, donde moran diversos encuentros que fueron transcritos. En el proceso de interpretación hermenéutica se debe considerar la siguiente metódica:

Leer varias veces el texto del relato, a esta actividad Desmarais (2009) la determina como "lecturas flotantes/ fluctuantes" (p.48). Es decir, la lectura que se realiza en reiteradas ocasiones desde el principio del texto hasta el fin, también a este paso, se le conoce como marcas guías a lo que Moreno (2007) explica: "la palabra marca conlleva a un cierto sentido de fijeza y le hemos añadido la palabra guía, unidas las dos con un guion para indicar su constitución en un solo vocablo" (p.37). Igualmente resalta que las marcas guías son: "señales de posibles significados organizadores que a lo largo de toda la historia puede convertirse en claves de comprensión del sentido disperso en ella y del núcleo frontal generante de todo el sentido y el significado" (p.37).

En efecto, las marcas guías consisten en leer línea a línea de manera profunda y en repetidas ocasiones para poder identificar el sentido y significados emergente de los relatos; desde este momento, se da apertura a la interpretación hermenéutica para captar los hechos impresos en las palabras, gestos y acciones, es decir, toda la realidad con sus matices. De acuerdo a Ferrarotti (2007), la lectura no se hace para adquirir información de un tema, para este autor es imprescindible que el texto sea habitado, que es igual a vivir dentro del él o involucrarse con entrega en la revisión profunda de cada una de las frases expresadas por el historiador.

Luego, se procede a descomponer el texto en unidades de significados que encierran un tema, dicho proceso conduce a la creación de categorías (Desmarais, 2009). Las categorías consisten en analizar el todo y clasificar en partes dotadas de significados, los principales elementos extraídos del mismo (Martínez, 2006); de esta manera, surgen categorías que permiten develar la realidad de los sujetos estudiados por medio de temas claves interconectados entre sí; cada categoría sugiere una subcategoría que consiste en la descomposición de la primera en términos de sus características y dimensiones, considerando las interrogantes cuándo, por qué, dónde y cómo. (Strauss y Corbin, 2002).

Desde la perspectiva de Moreno (2006), este paso se denomina "sentidos" y nacen de las marcas guías, definiendo sentidos como: "el último acontecimiento que da cuenta de la vida en ejercicio y que se explica no por otro acontecimiento sino por el hecho mismo de acontecer y acontecer así" (p.26). Pues bien, todo hecho tiene un sentido y revela un significado que pertenece a la realidad misma y es el resultado de los relatos. Una vez, que se haya realizado el proceso de lectura de cada línea y esta actitud sea conducida por la hermenéutica, se constituirán los sentidos que De Caires (2009), señala como bloques de sentidos:

Esto es, segmentos en los que queda dividido la narración y que se encuentran cargados de sentido y significado, ello se debe a que en los mismos se condensan toda una comprensión de significados a través de una o varias marcas-guías y de las que empiezan a elaborarse construcciones interpretativas que no son más que darle palabra a los significados profundos que son develados (p.32). 
De aquí la importancia de la interpretación, ya que permite descubrir los significados arraigados a la narración de la experiencia de los protagonistas con todas sus manifestaciones. Significa entonces, que las marcas guías y los bloques de sentido son técnicas de interpretación sistemáticas dirigidas a descubrir la esencia del fenómeno. Este paso, también es conocido por Ferarrotti (2007), como áreas problemáticas, quien señala que "una historia de vida puede concernir la socialización primaria (familia, escuela), la esfera sexual, el trabajo y la posibilidad de hallar una ocupación, como se dice, "creativa", los vínculos con la pareja y con los hijos" (p.108). En consecuencia, toda una vida desde sus diversas estructuras complejas o desde los distintos planos como lo específica Moreno. Por lo tanto, un investigador puede hallar en la interpretación de la historia-de-vida tres planos de profundidad o significación, a saber, Moreno, (2006):

- El plano personal: Este plano está involucra por los datos y significados perteneciente al individuo, es decir su personalidad, sus rasgos de carácter etc. Es el plano de lo psicológico, en el cual se efectúan diagnósticos educativos y psicológicos.

- El plano Grupal: Este plano se refiere a los diferentes modos de-vida que tiene una persona de acuerdo a su membresía a diversos grupos.

- El plano del mundo-de-vida: Es totalidad de vida, donde el hacer primario es el origen de los significados. Entiéndase como el ejercicio del vivir común de todos los sujetos representado en el convivir.

\section{Relevancia de los Significados y Símbolos en el Proceso de Interpretación}

Los significados y símbolos son los complejos culturales que se generan de las prácticas de vida comunes en una sociedad. Cuando éstas son asumidas por todos los integrantes de un grupo, se establecen como costumbres, valores y representaciones sociales que los caracterizan (Moreno, 2006).

La persona que narra su historia de vida no tiene dominio en los significados, ya que se manifiestan en toda su vida y en el momento del relato de la siguiente manera: "en el lenguaje, en la organización, en el ritmo de la narración, en la veracidad y falsedad consciente o inconsciente de lo narrado, en los gestos, en las frases o palabras significativas presentes en el discurso" (Moreno, 2006, p.25). En resumen, son los elementos que se toman en consideración para el análisis de una realidad, tal cual como la expresa el historiador; en esta naturalidad germina lo real, lo originario y lo puro que pertenece al protagonista de la historia (sujeto investigado).

\section{Conclusión}

Por medio de la investigación documental realizada, se puede concluir que la historia de vida es un método de investigación, derivado del enfoque biográfico que, a su vez, responde a la investigación cualitativa. El método de historia de vida constituye una perspectiva fenomenológica, ya que se fundamenta en la comprensión de la vida de un sujeto, tal como éste la experimenta en un espacio y tiempo determinado. Desde la diversidad de pensamiento, algunos autores califican la historia de vida como técnica o herramienta que, por sí misma no puede generar un proceso riguroso para alcanzar el conocimiento científico.

Sin embargo, se demuestra en el estudio que la historia de vida es un método autónomo de investigación porque demanda un modo sistemático para interpretar y comprender todo un 
entramado social, inmerso en los significados que cada historiador expresa en sus relatos. El trabajo del investigador radica en descubrir los diversos elementos subyacentes en el texto (historia de vida transcrita), a través de la hermenéutica, la cual en la práctica se evidencia en todo el proceso interpretativo que parte desde el primer contacto con los de los protagonistas, atraviesa la producción de la historia y culmina con la comprensión profunda de la realidad que envuelve la complejidad humana.

\section{Bibliografía}

Arias, F. (2012). El proyecto de investigación. Caracas, Venezuela: Editorial Episteme.

Bertaux, D. (2005) Los relatos de vida. Perspectiva etnosociológica. Barcelona, España: Bellaterra.

Bertaux, D. (1999). El enfoque biográfico, su validez metodológica, sus potencialidades. Proposiciones, 29(1), 1-23. Recuperado de http://www.sitiosur.cl/r.php?id=436Bisquerra, R. (2004). Metodología de la investigación educativa. Madrid, España: Editorial Muralla, S. A.

Bisquerra, R. (2004). Metodología de la investigación educativa. Madrid, España: Editorial Muralla, S. A.

Chárriez, M. (2012). Historias de vida: Una metodología de investigación cualitativa. Griot, 5(1), 50$67 . \quad$ Recuperado de http://ibdigital.uib.es/greenstone/collect/portal_social/index/assoc/miso1098/9_002.dir/miso 10989_002.pdf

Chárriez, M. (2012). Historias de vida: Una metodología de investigación cualitativa. Griot, 5(1), 5067. http://ibdigital.uib.es/greenstone/collect/portal_social/index/assoc/miso1098/9_002.dir/miso 10989_002.pdf

Cornejo, M., Mendoza, F., y Rojas, R. (2008). La investigación con relatos de vida: Pistas y opciones del diseño metodológico. Psykhe, 17(1), $29-39 . \quad$ Recuperado de http://www.scielo.cl/pdf/psykhe/v17n1/art04.pdf

Cornejo, M. (2006). El enfoque biográfico: trayectorias, desarrollos teóricos y perspectivas. Psykhe, 14(1), 95 - 106. Recuperado de http://www.redalyc.org/articulo.oa?id=96715108.

De Caires, E. (2009). La praxis concreta de la orientación y la función social del orientador desde la realidad socio cultural del venezolano (trabajo de ascenso). Universidad de Carabobo, Valencia, Venezuela

Desmarais, D. (2009). El enfoque biográfico. Cuestiones Pedagógicas, 20(17), 27-54. Recuperado de http://hdl.handle.net/11441/54980.

Denzin, N. y Lincoln, Y. (2012). El campo de la investigación cualitativa. Barcelona, España: Editorial Gedisa,

Ferrarotti, F. (2007). Las historias de vida como método. Convergencia, 14(44), 15 - 40. Recuperado de http://www.redalyc.org/articulo.oa? $i d=10504402$. 
Ferrarotti, F. (1990). La historia y lo cotidiano. Buenos aires - Argentina: Centro Editor de América Latina

Granados, L., Alvarado, S. y Carmona, J. (2017). Narrativas y resiliencia. Las historias de vida como mediación metodológica para reconstruir la existencia herida. CES Psicología, 10(1), 1-20. Recuperado de http://www.redalyc.org/articulo.oa?id=423550874001

Hernández, R., Fernández, C. y Baptista, P. (2014). Metodología de la investigación. México D. F., México: McGraw-Hill.

Mallimaci, F. y Giménez, V. (2006). Historia de vida y métodos biográficos. En I. Vasilachis de Gialdino (Ed.), Estrategias de investigación cualitativa. Barcelona, España: Editorial Gedisa.

Martínez, M. (2006). Comportamiento Humano. Nuevos métodos de investigación. México, D. F., México: Trillas.

Moreno, A. (2007). Y Salimos a matar gente: Investigación sobre el delincuente venezolano violento de origen popular, volumen I. Maracaibo, Venezuela: Universidad del Zulia.

Moreno, A. (2002). "Historias de vida" e Investigación. Caracas, Venezuela: Centro de investigaciones Populares.

Moreno, A., Brand, J., Campos, A., Navarro, R., Pérez, M., Rodríguez, W., y Varela, Y. (1998). Historia-de-vida de Felicia Valera. Caracas, Venezuela: Conicit.

Palella, S. y Martins, F. (2012). Metodología de la investigación cuantitativa. Caracas, Venezuela: FEDUPEL.

Córdova, V. (1990). Historias de vida: Una metodología alternativa para Ciencias Sociales. Caracas, Venezuela: Fondo Editorial Tropykos.

Pujadas, J. (2000). El método biográfico y los géneros de la memoria. Revista de Antropología Social, 9(1), 127-158 Recuperado de http://comisionporlamemoria.net/bibliografia2012/metodologia/Pujadas.pdf

Rodríguez, W. (2003). Las historias-de-vida. Conciencia Activa, 21(2),113-140. Recuperado de https://caredu.files.wordpress.com/2010/05/historias-de-vida.pdf

Sanz, A. (2005). El método biográfico en investigación social: Potencialidades y limitaciones de las fuentes orales y los documentos personales. Asclepio, 57(1), 99-115. Recuperado de http://dx.doi.org/10.3989/asclepio.2005.v57.i1.32

Strauss, A. y Corbin, J. (2002). Bases de la investigación cualitativa. Tecinas y procedimientos para desarrollar la teoría fundamentada. Medellín, Colombia: Universidad de Antioquia.

Taylor, S. y Bogdan, R. (1987). Introducción a los métodos cualitativos de investigación. Barcelona, España: Paidós. 